\title{
CONTROLE DE NÍVEL EM TANQUE ATRAVÉS DA MESCLA ENTRE SCILAB E ARDUINO BASEADO NO CONTROLE PI
}

\author{
Cássio Rodolfo Aveiro da Silva*; Cid Marcos Gonçalves Andrade; Alisson Cocci de Souza; Victor \\ Cesar Panuci; Wagner André dos Santos Conceição; Mônica Ronobo Coutinho. \\ *Universidade Estadual de Maringá - UEM - PR, crasilva2@uem.br
}

\section{RESUMO}

Para um sistema de controle é necessário que haja um ou mais controladores. Dentre os mais utilizados no ramo industrial, estão os controladores PID e PI. Criado pelas empresas francesas INRIA e ENPEC o Scilab caracteriza-se como um software de código livre, não apresentando custo para sua aquisição, além de contar com uma ferramenta integrada chamada Xcos, destinada à modelagem e simulação. Para a união com o Scilab, está o Arduino. Tal mescla pode ser empregada em qualquer sistema de controle. Este trabalho objetivou, desenvolver um controle de nível em tanque baseado no controlador PI através da união entre Scilab e Arduino. Um modelo fenomenológico foi desenvolvido com base na malha de controle fechada do processo. Para a sintonia dos parâmetros do controlador foi utilizado o método ISE executado por meio de um código de programação desenvolvido no Scilab. Os parâmetros encontrados foram testados em uma bancada experimental. Por meio de um diagrama de blocos do método ISE foram obtidos valores satisfatórios para os parâmetros proporcional e integral ratificados por meio dos testes realizados no módulo experimental indicando que o controle de nível foi alcançado.

Palavras-chave: Scilab, Arduino, controlador PI, ISE, controle de nível.

\section{INTRODUÇÃO}

Ao se unir dispositivos distintos com a finalidade de obter uma resposta específica para um dado processo em questão, surge o que geralmente é chamado de sistema de controle [1]. Realizar um processo de controle, refere-se a manter uma determinada variável (grandeza ou condição) em um valor desejado - comumente chamado de valor de referência ou simplesmente "Referência" - ou o mais próximo possível deste [2].

Para que seja possível o desenvolvimento de um sistema de controle, é necessário a utilização de um controlador. Dentre os diversos e distintos controladores disponíveis, os controladores Proporcional (P), Proporcional e Derivativo (PD), Proporcional e Integral (PI) e Proporcional, Integral e Derivativo (PID) são os que mais se destacam e são mais utilizados em diversos ramos da engenharia, sobretudo no ramo industrial [2]. Isto, em virtude da facilidade e simplicidade de serem implementados, além de uma grande quantidade de estudos científicos disponíveis no que tange os métodos para o ajuste e sintonia de seus parâmetros [3].

Para se proceder com a implementação de um sistema de controle, faz-se necessário o uso de softwares e hardwares específicos. Os softwares são utilizados como forma de se realizar uma simulação do comportamento do processo o qual de deseja controlar, permitindo a criação do modelo fenomenológico - baseado em uma modelagem matemática do processo - e a sintonia dos parâmetros do controlador [4].

$\mathrm{Na}$ engenharia de controle, nomes como Matlab ${ }^{\circledR}$, LabView $^{\mathrm{TM}}$, Maple $^{\circledR}$, Excel $^{\circledR} \mathrm{e}$ Scilab são amplamente conhecidos e utilizados [5]. Os três primeiros, apesar de suas grandes capacidades computacionais, apresentam uma aquisição de licença para utilização muito custosa. Por outro lado, o software Scilab, desenvolvido pelas empresas francesas INRIA e ENPEC como uma alternativa ao Matlab ${ }^{\circledR}$ (com poder computacional que vai desde o cálculo numérico ao processamento de sinal e design de sistemas de controle) é disponibilizado como software de licença livre, permitindo ao usuário ter 
acesso ao seu código fonte e alterá-lo da melhor forma que se adapte às suas necessidades, tudo isso de forma totalmente gratuita. O software apresenta também uma ferramenta de modelagem e simulação denominada Xcos que oferece uma interface gráfica capaz de editar modelos e interligar os blocos que representam por exemplo, as funções de um sistema de controle [6].

Dentre os hardwares utilizados em sistemas de controle, tem-se o Arduino. Tratase de uma plataforma de código aberto baseada em software e hardware de baixo custo e de simples utilização, com aplicação sobretudo em projetos eletrônicos de aquisição de dados, monitoramento e controle [7].

Neste contexto, o trabalho aqui apresentado abrange uma das aplicações mais comuns dos sistemas de controle; o controle do nível de líquidos em tanques industriais [8].

Isto posto, o objetivo geral deste trabalhado é desenvolver um modelo fenomenológico de um sistema de controle de nível através de um controlador PI por meio da união entre o Scilab e a plataforma Arduino e testar tal controle em uma bancada experimental.

\section{EXPERIMENTAL}

Para a realização deste trabalho, foi considerado um processo no qual um tanque (de $30 \mathrm{~cm}$ de altura e área transversal de $660,57 \mathrm{~cm}^{2}$ ) deve ter sua altura de nível de líquido controlada de acordo com um valor de Referência pré-determinado.

O primeiro passo para o desenvolvimento do sistema de controle foi modelar matematicamente o processo. Foi estabelecido o modelo (Fig. 1) composto por: uma vazão de entrada $\left(\mathrm{q}_{0}\right)$; uma vazão de saída $\left(\mathrm{q}_{1}\right)$ com passagem pela válvula reguladora de fluxo - Variável Manipulada.

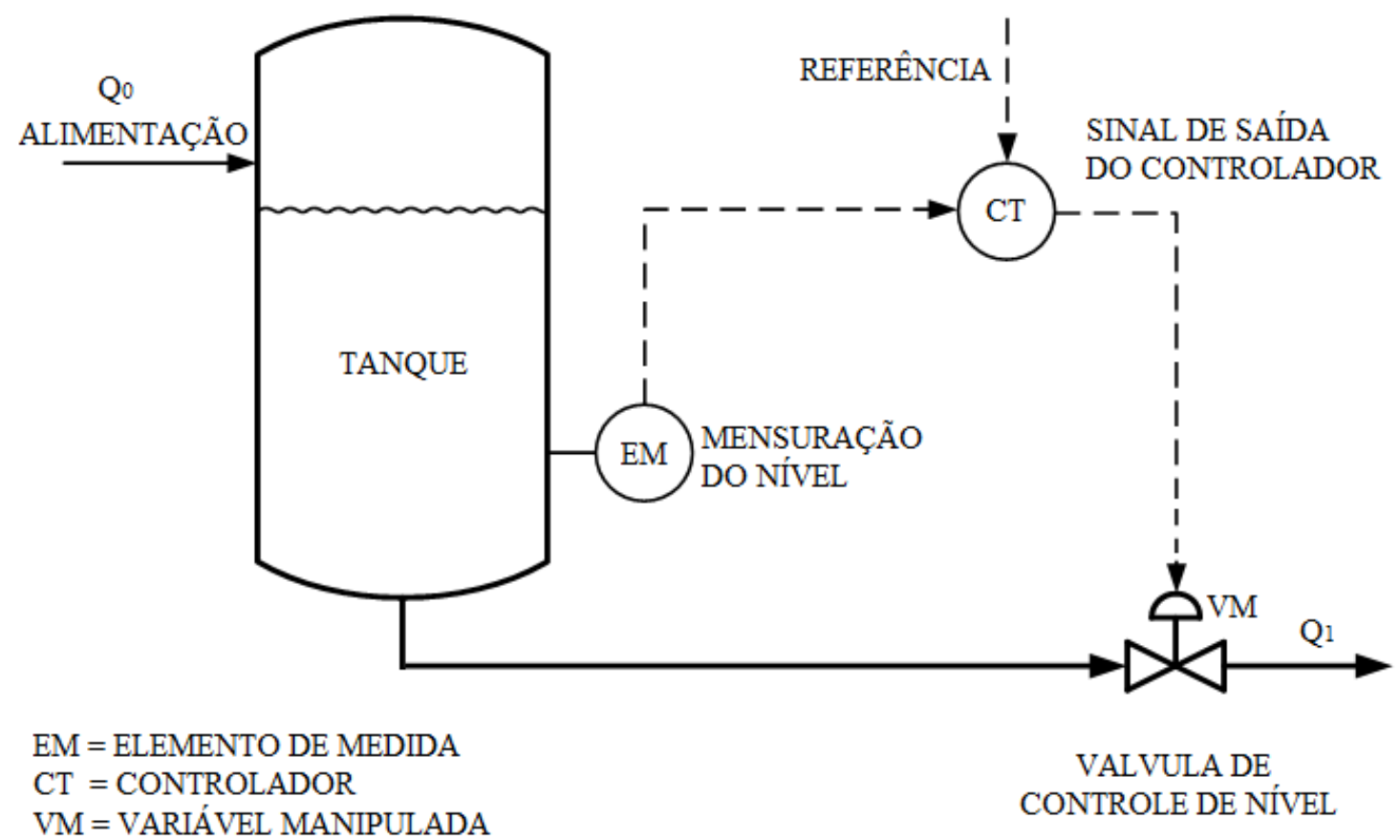

Fig. 1 - Representação do fluxo de líquido em um tanque. Fonte: Autor, 2018. 
Com o balanço de massa referente ao acumulo de líquido no tanque obteve-se a seguinte expressão [9]:

$\mathrm{A}_{1} \frac{\mathrm{d}\left(\mathrm{h}_{1}\right)}{\mathrm{dt}}=\mathrm{q}_{0}-\mathrm{q}_{1}$

onde $\mathrm{A}_{1}$ é a área transversal do tanque e $\mathrm{h}_{1}$ é sua altura referente ao nível de líquido. Por meio de manipulações algébricas e de uma relação com o estado estacionário do sistema, a Eq. 1 torna-se:

$\mathrm{H}_{1}(\mathrm{~s})=\left[\mathrm{Q}_{0}(\mathrm{~s})-\mathrm{Q}_{1}(\mathrm{~s})\right] * \frac{1}{\mathrm{~A}_{1} \mathrm{~s}}$

escrita em termos da Transformada de Laplace representando pois, a função transferência para o fluxo de líquido no tanque. $\mathrm{Q}_{0}=\mathrm{q}_{0}-\mathrm{q}_{0 \text { (estacionário) }}, \mathrm{Q}_{1}=\mathrm{q}_{1}-\mathrm{q}_{1 \text { (estacionário) }}$ e $\mathrm{H}_{1}=\mathrm{h}_{1}-\mathrm{h}_{1 \text { (estacionário) representam as variáveis de desvio do sistema. }}$

De acordo com o objetivo proposto, o controlador utilizado para o sistema de controle do nível do tanque foi o controlador PI definido pela soma das ações Proporcional e Integral do tradicional controlador PID em um mesmo decorrer de tempo no processo. O sinal de saída do controlador é definido matematicamente por:

$u(t)=K_{p} * E(t)+K i \int E(t) d t+K_{d} \frac{d E(t)}{d t}$

onde $\mathrm{K}_{\mathrm{p}}$ é o ganho proporcional, $\mathrm{K}_{\mathrm{i}}$ é o ganho integral e $\mathrm{K}_{\mathrm{d}}$ é o ganho derivativo do controlador. Este último termo, é zerado afim de que apenas o controle PI seja aplicado.

Com base na modelagem matemática do sistema e do tipo de controlador escolhido para o processo, uma malha de controle caracterizada por um sistema de feedback foi desenvolvida como forma de representar o sistema de controle (Fig. 2).

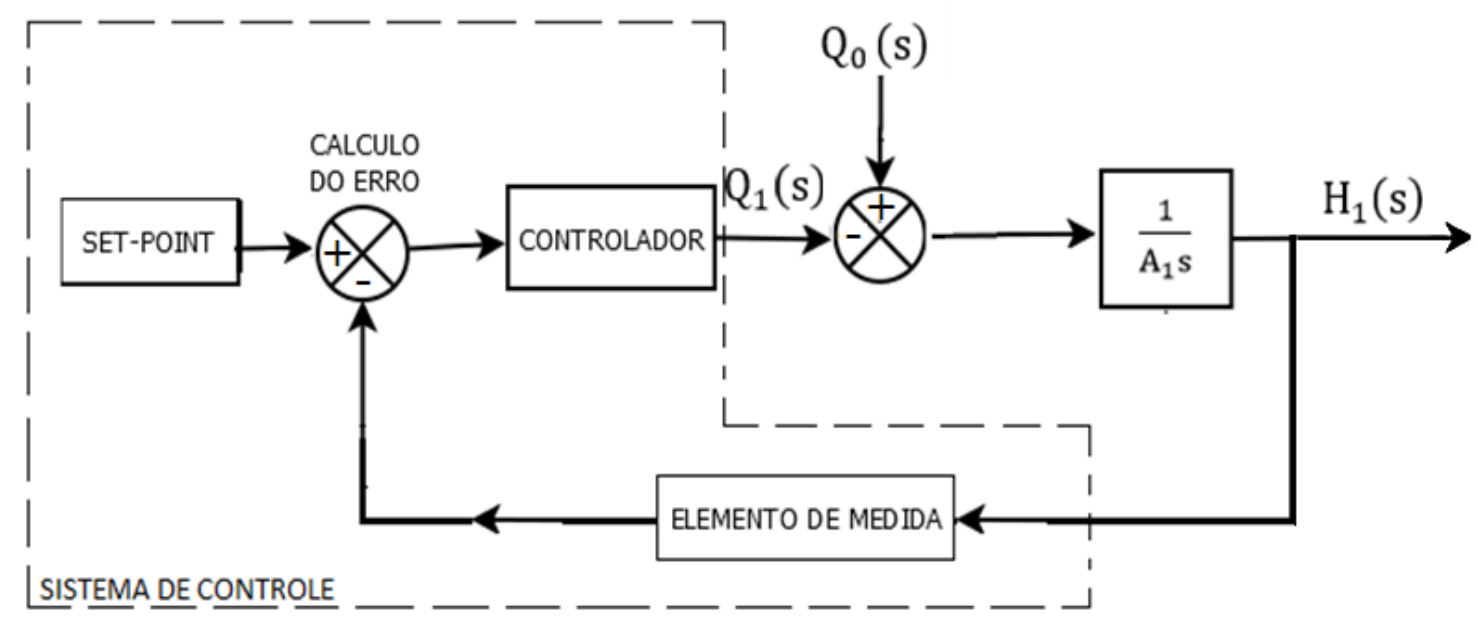

Fig. 2 - Representação da malha de controle do processo. Fonte: Autor, 2018.

Uma vez criada a malha de controle, foi desenvolvido um diagrama de bloco no ambiente Scilab em sua versão 5.5.0 através da ferramenta Xcos (em formato *.zcos). Por meio deste, foi possível implementar um bloco referente ao controlador PID (aplicado em forma de controle PI). Além disso, adicionou-se ao diagrama, um bloco para retardo da 
ação da variável manipulada. Foi estabelecido que a cada cinco segundos, dez valores de nível serão medidos e destes calculada a média. Logo, a ação da variável manipulada ficará condicionada a este período (tempo morto). Isto se fez necessário para que as variações na leitura executada pelo sensor - no módulo experimental que será abordado na sequência - não interferiram significativamente no processo de controle.

Para a implementação do controlador no sistema de tal modo a proporcionar a resposta desejada para a variável controlada (nível de líquido), a sintonia de seus parâmetros se fez necessária.

Existem inúmeras técnicas e métodos para que a sintonia de parâmetros proporcional, integral e derivativo seja alcançada. Para o caso apresentado neste trabalho, o método de sintonia escolhido foi o Método da Integral do Erro Quadrático - Integral of Squared Errors (ISE) definido como:

$\operatorname{ISE}=\int_{0}^{t} E^{2}(t) d t$

onde E é o Erro e o limite superior da integral é definido como suficientemente grande para que o método seja aplicado não só ao período transitório como também ao período em regime estacionário do sistema.

$\mathrm{O}$ processo de fluxo de líquido em tanques apresenta uma rápida resposta, porém sem apresentar oscilações excessivas o que justifica a utilização do Método ISE [10]. O método visa obter valores para os parâmetros do controlador que proporcionem curvas resposta satisfatórias para o controle. Assim sendo, como proposta metodológica estabelecida e afim de evitar manobras do tipo "tentativa e erro", foi desenvolvido um código de programação (em formato *.sci) no ambiente de script do Scilab baseado em uma "função ISE" presente na biblioteca interna do software. Tal código, permite abrir o diagrama de bloco criado no Xcos, adicionar inicialmente e em forma de "chute" inicial, valores para $\mathrm{K}_{\mathrm{p}}$ e $\mathrm{K}_{\mathrm{i}}$. A identificação do sistema que representa o processo (submetido a uma variação na carga de entrada) e a sintonia dos parâmetros é executada. Por meio da definição do número de iterações e do erro aceitável para o sistema de controle, é realizada um laço condicional. Se a sintonia apresentar um valor de ISE aceitável de acordo com o que foi definido, o programa se encerra e apresenta os melhores valores dos parâmetros encontrados de forma tanto numérica como gráfica. Caso contrário, o programa retorna ao ponto onde novos valores de $\mathrm{K}_{\mathrm{p}}$ e $\mathrm{K}_{\mathrm{i}}$ são implementados e uma nova iteração acontece em um processo que se repete até a condição descrita ser alcançada.

Com a sintonia dos parâmetros obtida, uma simulação por meio da ferramenta Xcos foi realizada, afim de apresentar a resposta do sistema de controle submetido a perturbações e variações no valor de referência. O tempo de simulação foi definido em 600 segundos (10 minutos) com o valor do nível inicialmente em $20 \mathrm{~cm}$ submetido a uma variação do valor de referência inicialmente em $25 \mathrm{~cm}$ e depois em $18 \mathrm{~cm}$.

Por fim foi confeccionado uma bancada experimental (Fig. 3), na qual a variável manipulada é acionada por um motor de passo (modelo NEMA17PM-K342B) em conjunto com o drive de controle (Ponte H L298N). Para a leitura do nível foi escolhido um sensor ultrassônico (modelo HC-SR04) com precisão de $0,3 \mathrm{~cm}$. 


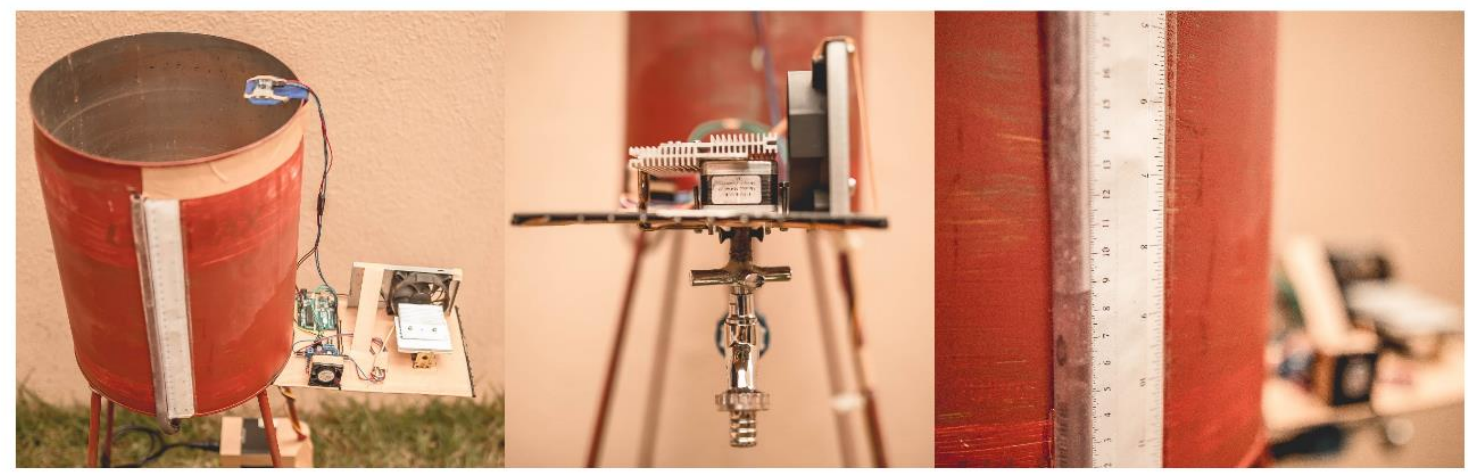

Fig. 3 - Bancada experimental. Fonte: Autor, 2018

O sensor capta o valor do nível de líquido no tanque e envia um sinal à plataforma Arduino. Esta por sua vez, está programada para realizar o processo de controle baseado no controlador PI de acordo com o valor de referência que lhe é estabelecido. O Arduino então, envia um sinal de controle (convertido em número de passos) ao motor de passos que então, atua sobre a variável manipulada aumentando ou diminuindo a vazão de saída do tanque com o objetivo de reduzir o erro entre a referência e o valor do nível (variável controlada).

Como forma de completar o trabalho, testes referentes aos parâmetros sintonizados do controlador, foram realizados na bancada experimental e os resultados discutidos.

\section{RESULTADOS E DISCUSSÕES} (Fig. 4).

Como resultado preliminar, foi obtido o diagrama de blocos no ambiente Xcos

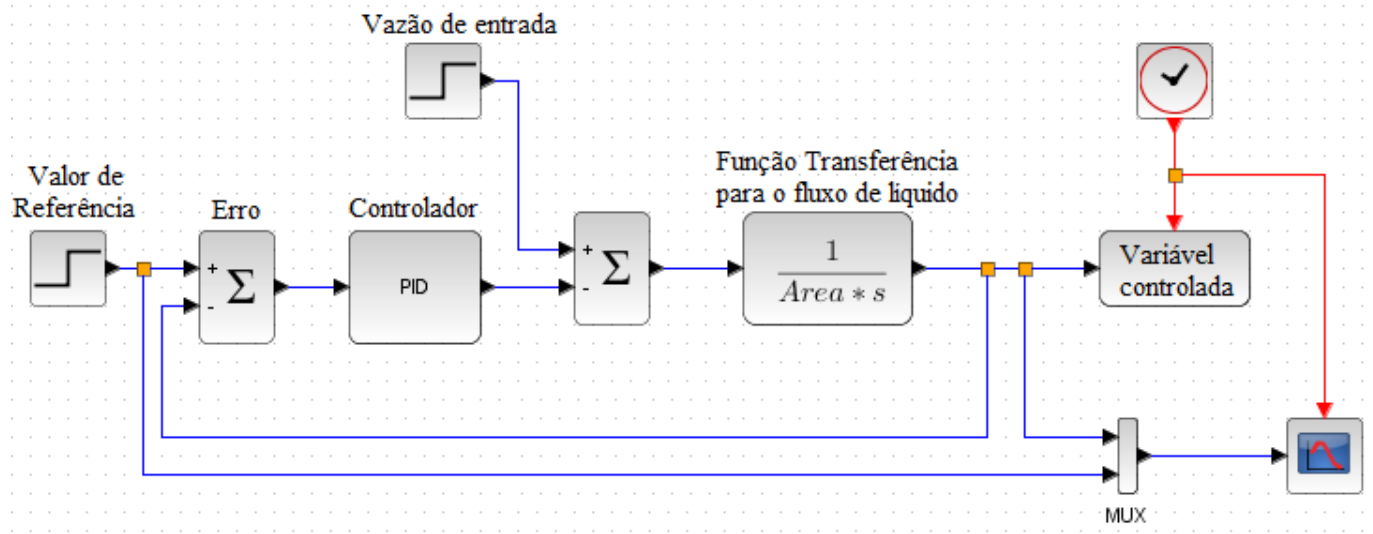

Fig. 4 - Representação do modelo fenomenológico do sistema de controle. Fonte: Autor, 2018

Com base deste diagrama, a sintonia dos parâmetros PI foi facilitada e alcançada pelo método ISE. Além disso, a simulação da resposta do sistema de controle também foi desenvolvida sobre tal modelo fenomenológico.

Com relação a execução do código de sintonia dos parâmetros do controlador PI, uma curva reposta para o controle PI foi obtida (Fig. 5). 


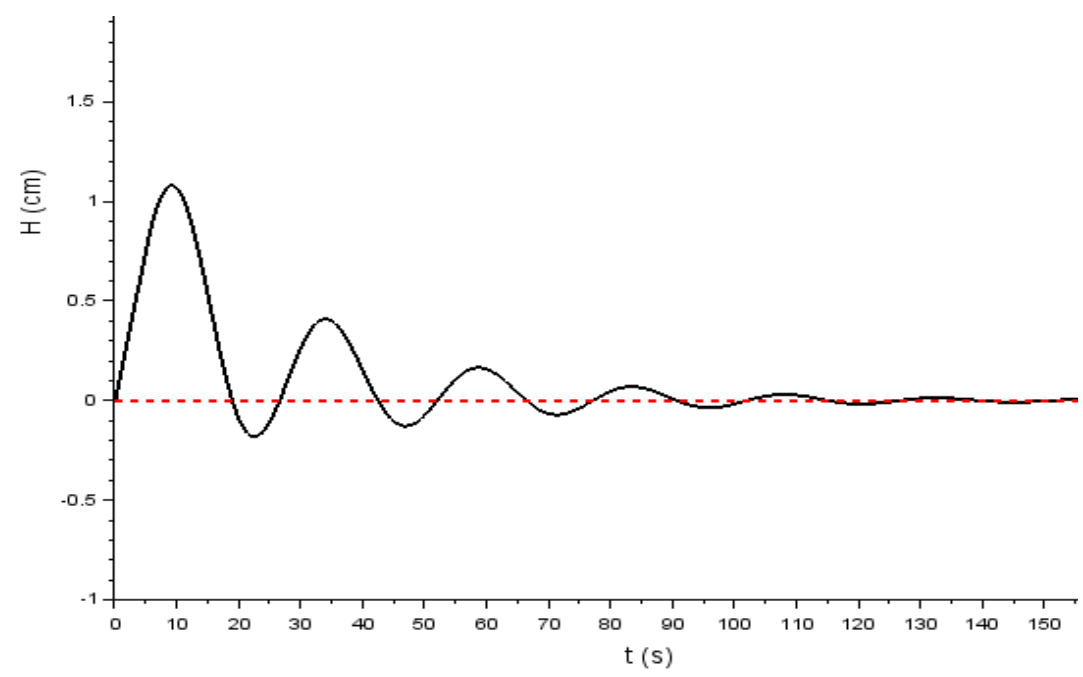

Figura 5 - Representação da curva resposta do sistema de controle PI com perturbação na carga. Fonte: Autor, 2018.

Com os valores dos parâmetros $\mathrm{K}_{\mathrm{p}}$, $\mathrm{K}_{\mathrm{i}}$ e $\mathrm{K}_{\mathrm{d}}$ iguais a -146,81; -6,39; e, zero, respectivamente, pode-se observar que houve a ocorrência de 7 (sete) picos (positivos e negativos) antes do sistema entrar em "estabilidade" em aproximadamente de 95,8 segundos após a perturbação carga. A ocorrência de oscilação inicial, é justificada vez que o sistema de nível de tanque proposto apresenta dimensões relativamente pequenas a nível industrial e a vazão máxima de saída é baixa, onde o controle apresenta uma reposta mais lenta em função disto. Porém o número de oscilações é considerado baixo e satisfatório. Uma resposta com oscilações em excesso, corresponderia a uma atuação da válvula de controle (variável manipulada) excessiva e consequentemente sobrecarga. Neste controle do tipo PI o valor do ISE de 11,60.

Com valores considerados satisfatórios para o sistema de controle, a simulação do processo submetido a perturbações no valor de referência foi realizada no modelo fenomenológico e posteriormente testados os parâmetros obtidos na bancada experimental conforme estabelecido anteriormente. Assim o seguinte resultado gráfico foi obtido (Fig. 6)

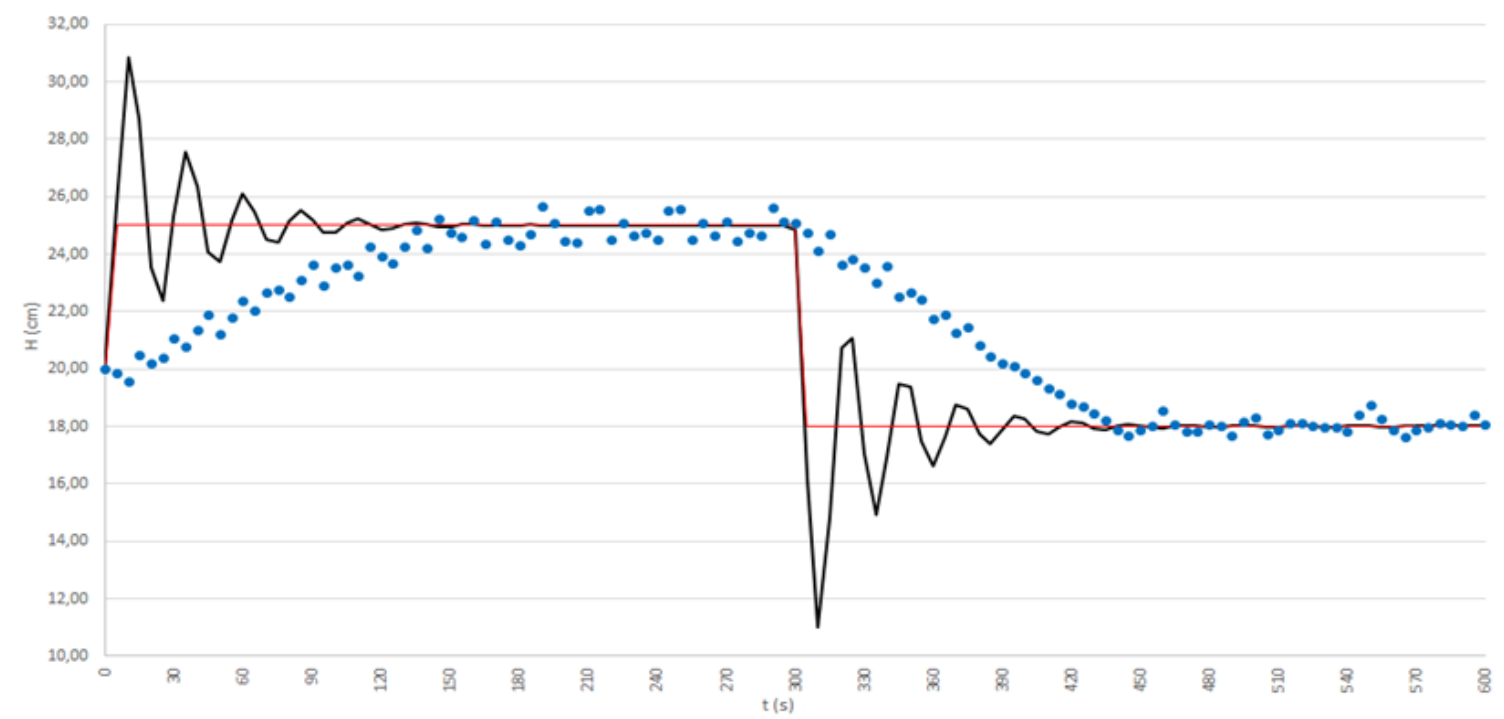

Fig. 6 - Representação das curvas respostas do sistema de controle PI com perturbações na referência. Fonte: Autor, 2018. 
O comportamento do sistema na bancada experimental (pontos azuis no Fig. 6) mostrou-se satisfatório e coerente com a curva resposta do modelo fenomenológico. A estabilidade do sistema para a primeira perturbação na referência foi alcançada, tanto pela simulação quanto pela resposta experimental, em um tempo estimado de 150 segundos (dois minutos e meio). Já para segunda perturbação, foi repetido o tempo para estabilização do sistema em 150 segundos. A suposta "incoerência" no início do controle com a não ocorrência de oscilações por parte do módulo experimental, apresenta um controle consideravelmente melhor sem sobrecarga da variável manipula. Tal fato se deve às limitações físicas da válvula utilizada, apresentando uma vazão máxima de $54,8 \mathrm{~cm}^{3} / \mathrm{s}$ e uma vazão mínima de zero, não consideradas pelo modelo fenomenológico.

\section{CONCLUSÃO}

O controle do nível foi realizado com base no controlador PI por meio da mescla entre o software Scilab e a plataforma Arduino. Isto foi possível em virtude do desenvolvimento de um diagrama de blocos com o auxílio da ferramenta Xcos, o que possibilitou que o código de programação criado para o método ISE obtivesse de forma relativamente simples os parâmetros proporcional e integral do controlador. O método ISE por sua vez, em função das características de resposta do processo em questão, se mostrou eficaz obtendo, como já citado, valores de parâmetros que ao serem testados experimentalmente, apresentaram uma resposta melhor que a própria curva resposta do modelo fenomenológico.

Portanto, um controle realizado por meio da união entre Scilab e Arduino combinada com uma representação em diagrama de blocos adequada do processo e com um método eficiente de sintonia, tornou-se totalmente alcançável e com excelentes resultados.

\section{REFERÊNCIAS}

[1] KITTUR, J. Enahncing the controller design skills in the course linear control systems. Journal of Engineering Education Transformations. Special Issue. 2018.

[2] SIMONELLI, G.; MAI, B. F.; VICHELO, D. R. S.; MARCHI, H. F.; CARVALHO, R. F. Simulação do controle de uma coluna de destilação descontínua utilizando o Scilab. Engevista. v. 19, n. 2, p. 498-519, 2017.

[3] URREGO, J. A. R.; RESTREPO, N. L. P. Aplicación de Diseño, simulación, identificación de sistemas e implementación de controladores PID - DIGITROL. Revista Politécnica. v. 12, n. 23, p. 27-37, 2016.

[4] BARRETO, L.; ESCOBAR, A. Aspectos prácticos de implementación em compensadores PID para control de posición de un automóvil. Ingenium. v. 15, n. 29, p. 148-162, 2014.

[5] LARSON, R.; FARBER, B. Estatística aplicada. Tradução de Luciane Ferreira. $4^{\mathrm{a}}$. ed. São Paulo: Pearson, 2010.

[6] SANJUAN, J. J. V.; PÉREZ, L. I. O.; MENDOZA, E. Y. Scilab-Arduino: Diseño e implementación de sistemas de control. ResearchGate. 2015. 
[7] PANUCI, V. C. Análise teórica, numérica e experimental de um coletor solar de placas planas destinado ao aquecimento de ar. Dissertação (mestrado) -

Universidade Estadual de Maringá, Centro de Ciências Exatas, Departamento de Engenharia Mecânica, Programa de Pós-Graduação em Engenharia Mecânica, 2017.

[8] SILVA, J. T.; SILVA, J. T.; LIMA, G. F. Controle e monitoramento de nível utilizando plataforma open source Arduino. Revista INNOVER. v. 1, n. 4, 2014.

[9] MAYA, P. A.; LEONARDI, F. Controle Essencial. 2a. ed. São Paulo: Editora Prentice Hall, 2014.

[10] FERMINO, F. Estudo comparativo dos métodos de sintonia de controladores PID. Trabalho de Conclusão de Curso - TCC. Engenharia Elétrica com ênfase em Sistemas de Energia e Automação. Escola de Engenharia de São Carlos, Universidade de São Paulo. São Carlos - SP. 2014. 